\title{
FOSS Enhanced Microkernel-based LBS Platform
}

\author{
Shang Zhang, Ming Xiao \\ College of Computer and Information Technology \\ China Three Gorges University \\ Yichang, China \\ wetoo@163.com
}

\author{
Liufeng Tao \\ Faculty of Information Engineering \\ China University of Geosciences \\ Wuhan, China
}

\begin{abstract}
Geographical Information System (GIS) always play an integral role in information systems, but it comes with technology problem, such as low efficiency ,less flexible, no redundancy of existing GIS application configuration which have high entry cost. The aim of this paper is to presents a lightweight, efficient and scalable microkernel plug-in geospatial information application system and its implementation method for GIS in microkernel design and practice. In this paper a software model called Resource loading manager (RLM) is designed. Through the RLM efficient allocation of geographic information resources and security management could be achieved. Using existing cloud computing technology, GIS of spatial public service will impact the ordinary way of life, bring enormous business opportunities.
\end{abstract}

Keywords-FOSS; RLM; Microkernel; LBS; OSGIS.

\section{INTRODUCTION}

Spatial Information technology prompted the generations of Space-Air-Ground Integrated Earth Observation Network, a new spatial information technology field [1].The goal of this new technology of spatial information includes not only the institutions, the government, companies and other professional groups, but also the public. How to solve the massive integration of heterogeneous data management and storage, parallel spatial information processing and analyzing, optical service on demand and other technical needs, has driven scientists to focus on meeting the diverse users with different types of needs, providing the information service based on spatial, Which is considered to be one of the most promising applications of Geosciences.

Meanwhile, open source strategy is another way to promote developments within the Geospatial processing domain currently, especially for the ongoing development and research topic "cloud". The availability of Free and Open Source Software (FOSS) for Geospatial Information systems (GIS) have increased tremendously in recent years [3].

\section{BRIEF REVIEW OF OSGIS AND LBS}

\section{A. About FOSS}

Free/libre/open source software (FLOSS) or open source software (FOSS) is technically defined as software whose licenses grant the right to use, copy, study, change, and improve its design for any purpose to the users, and no further limitations or royalty payments need when redistributing either the original or modified program. This, to a certain

This paper is supported by the China Three Gorges University Science \& Technology Foundation program (No. 1114072). degree, prevents the users to set restrictions to other users. In order to make this possible, access to the source code is a necessary condition $[3,4]$.

\section{B. Brief of OSGIS}

GIS is becoming an important framework for understanding a dynamic world. More and more GIS have been applied in national economy and social life, they stand in need of the interoperability and reusability. A large commercial GIS industry has emerged to provide customers with software tools, training, and source data. The scope of GIS implementation is also extensive-from a desktop implementation to an enterprise operation investing significantly in data storage and processing assets. However, the cost of large deployments using commercial software can be prohibitive [2].

At the same time the limitations of traditional programming method with the interoperability, portability and data sharing became more obvious. In these cases, open source applications have an equal potential for large-scale implementation across a broad range of platforms, but they can be far more cost-effective and flexible [4]. OpenGIS (Open GIS Interoperability Specification) is coming to address the above issues, the specification defines a detailed criteria for GIS software developers.

To satisfy such trends of the information system, the open community has been developing the component-based Open GIS software system [3].The OGC (Open GIS Consortium) is an attempt to create open standards for geo-spatial data and systems that further the cause of interoperability. Component is a software pieces that can be assembled into various applications. For the sake of the reusability, a component-ware or distributed objects is a new approach to software development.

Software engineers will develop the product which can meet the requirement and inter-operate well, as long as strictly abide by the OpenGIS standards, that is, the product can achieve the data and function sharing between GISs.

\section{MICROKERNEL AND RLM}

In recent years, as the development of software systems in style of "component" and "hierarchical", micro-kernel technology has gradually been introduced into software design framework for the "decoupling possible relationships between 
components." The concept of Microkernel (micro kernel) was originally made for building microkernel operating system based on message passing mechanism, not for software architecture services [5]. But in the field of GIS, microkernelbased system has not yet been established. But the way of microkernel based system provided several benefits that traditional GIS platform don't have:

a) Easy to modify the existing similar software systems.

b) There is no need to develop new application systems to meet user changed needs;

A geospatial information support platform based on microkernel architecture technology which provides a lightweight runtime environment has been established in this paper, including open geographic information processing, remote sensing data processing and mutual integration process. In this case, not only a lot of software purchase costs can be saved, but also a variety of personalized geospatial information processing demand can be meet.[6].

\section{A. Resource Loading Manager}

In order to ensure the reasonable resource management, a geospatial information application system based on micro kernel technology is designed in this paper. Geospatial information resource loading manager (RLM) which used to load the application of the plug-in function module of program could realize the business logic of the application program. The structure of the RLM is shown in figure 1[8].

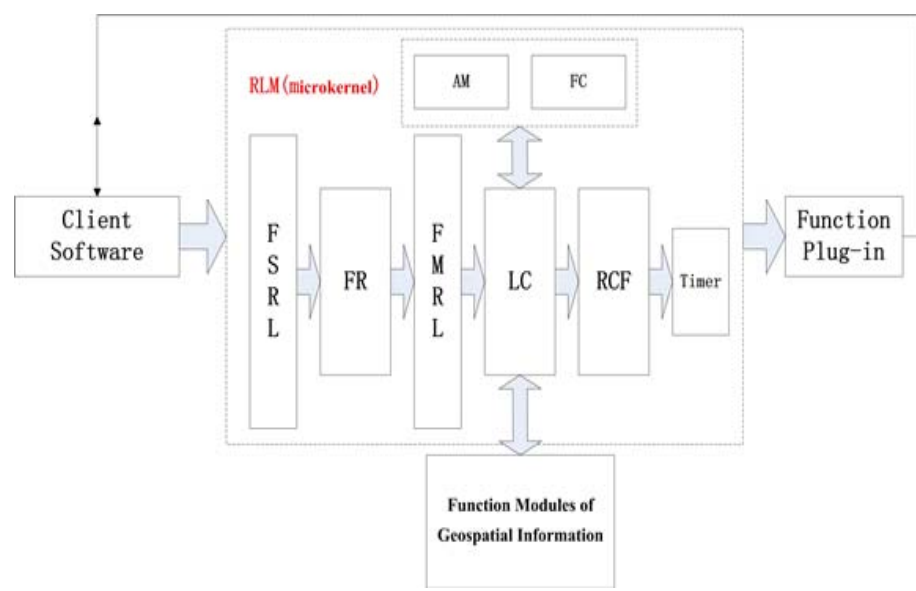

Figure 1. The structure of the RLM.

From Figure 1, FSRL (functional service requirements list) is used to count the resources required by the client software. The information includes at least: client software security level, the features of required resources, and the method of administration.

According to FSRL, FR (function resolver) puts the functional requirements of client software in accordance with the rules of the system function modules to subdivide, and builds functional modules list of requirements.

FMRL (functional modules requirements list) used to describe the function modules refined from the functional service requirements of client software.
LC (list counters) is used to allocate function modules according to FMRL.

RCF (resource configuration file) is used to identify all functional modules.

AM (access manager) is used to check the LC calls to functional modules permissions.

FC (function combiner) is used to package the function modules called by LC for plug-in, using for client software.

The Timer is used in one or more functional blocks occupied by other programs, and cannot be immediately called. The timer periodically detects the required permissions whether the function modules were released, and dynamically loaded them into the function plug-in when they were released.

\section{B. Implementation of RLM}

The basic idea of the method described above is: The process logic of programming system is implemented through a plug of expression of microkernel architecture. FR is used to decompose the functional requirements of client software into functional modules requirements. LC re-organize and dispatches modules, and through FC to provide plug-ins for client software. Furthermore, in order to ensure the timeliness, accuracy of function calls, we use RCF and AM to manage the resources. Meanwhile, the timer periodically detects the function module which unable to be used temporarily. When the function permits to release it, the FC immediately loads into the plug-in functionality for client software to use. In American English, commas, semi-/colons, periods, question and exclamation marks are located within quotation marks only when a complete thought or name is cited, such as a title or full quotation. When quotation marks are used, instead of a bold or italic typeface, to highlight a word or phrase, punctuation should appear outside of the quotation marks. A parenthetical phrase or statement at the end of a sentence is punctuated outside of the closing parenthesis (like this). (A parenthetical sentence is punctuated within the parentheses.)

\section{A Prototype GIS SERVICE Platform Design}

Data is the foundation of all applications and services. The uniqueness and accuracy are the important characteristics of a geographic entity, therefore, in this LBS platform (as in Figure 3) differences and ambiguity caused by the multiple data sources must be overcome. This platform in the GIS based service is to deal with all kinds of basic data, including the navigation electronic maps of all kinds of manufacturers, all kinds of industry data and remote sensing image data. GIS based service mainly has two parts, one is an online service support system, and another is a middle-ware system. The design, implementation, operation of these two parts require rigorous data standard and constraint service specification.

\section{A. Online Support Service System}

For online service support system, based on the data management module, which conduct the most basic data management, multi-source data fusion, data processing, thirdparty data service support and so on. The design is to build a 
quick compatibility and integration of a variety of data sources of standard geographic data management system.

In establishing such a unified geographic data management system, flexible scheduling and data dispatch module are needed. Due to the diversity of the LBS service and the growing demand for the industry, different service requirements for data format are also diverse. Through data scheduling and dispatch module, and according to the data content choice, the user can specify its own data format needs, and carry on the content data release, data integration and updating. Therefore, all data are standard unified and constant. The diversification is also the important guarantee of accurate GIS service. According to industry application and characteristics of the public service, while through the data access middle-ware and services middle-ware interface, the LBS platform can be customized.

The Nav service support module is a platform to support various types of online services, it mainly includes various navigation electronic map service functions, such as spatial analysis, buffer analysis, data scheduling, information processing, online service application, user management, and other functions. Those functions are designed to provide online function module for the user of third party, and also could for the middle-ware design and development, which can be composed for all kinds of navigation application.

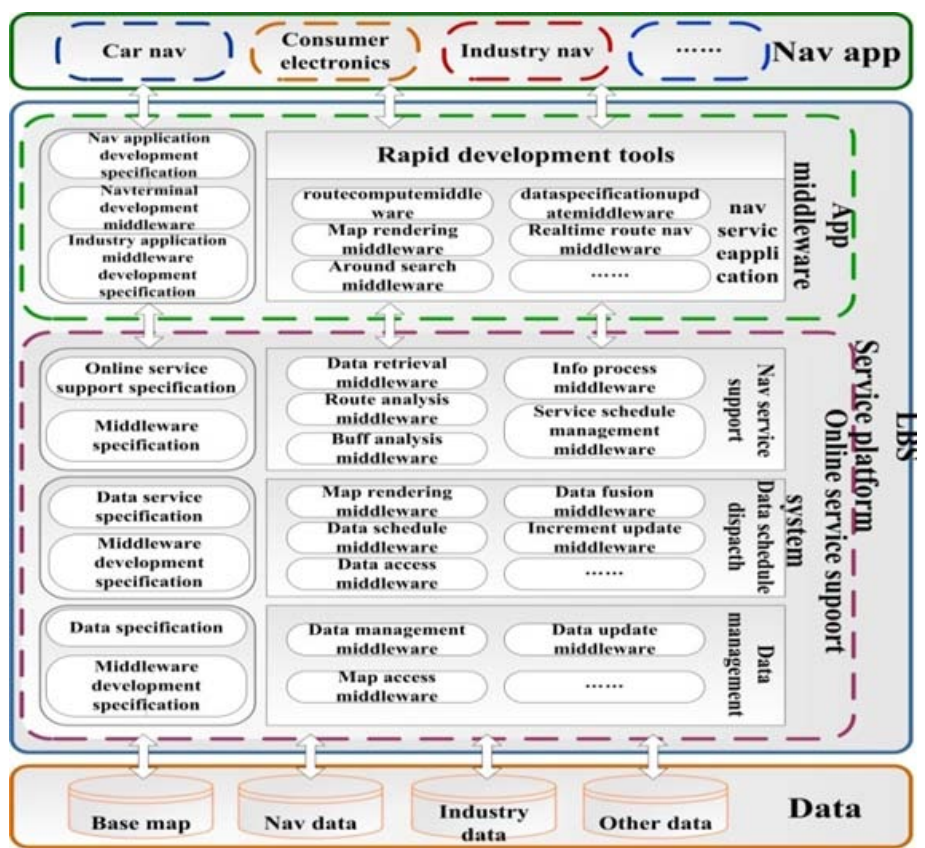

Figure 1. The architecture of GIS service platform

\section{B. The Middle-ware Platform}

Middle-ware system is the core of GIS service, which throughout the underlying data management to online services support, and the application development, middle-ware technology process.

The service platform composed of the various types of middle-ware. In order to achieve structure flexible and functional independence, function modules are based on middle-ware implementation, which are basement of the infrastructure of the services platform.

In the field of electronic map navigation application, middle-ware is the main form and content of navigation terminal development, their complementation are completely followed by the specification shown in the Firgure-1. In development, the use of middle-ware can have better classification of all kinds of functions and extensions, which can assist the user to build and deploy the GIS services.

Otherwise the rapid development tools are, moreover, providing an important feature of GIS platform, which increase the difficulty of software development, shorten the development cycle, and solve the problem of multiplication differences. The tool can support multiple mobile devices in terms of building type development; the development of the core functionality can be implemented directly by the middleware in the form of drag and drop. Rapid development tools make full use of the navigation terminal development, middleware and industry application which could improve the efficiency of software development, enhances its extensibility.

\section{CONCLUSION}

Spatial public service will also change the ordinary way of life, and bring enormous business opportunities. At present, the high costs of information sharing system embark the spatial public service. The development and application of FOSS or OSCIG based product will provide spatial information service at high efficiency and low cost.

Based on FOSS/OSGIS, the design of middle-ware GIS platform, which can absorb technological and scientific achievements in the field of information technology synchronously, could keep up with advanced concept of software architecture, so as to promote the development of spatial service technology and software.

Meanwhile microkernel technologies, which can absorb scientific and technological achievements in the field of information technology synchronously, keep up with advanced concept of software architecture, so as to promote the development of GIS technology and software.

At the same time, based on micro kernel technology, we build the atom granularity function module of management and automatic loading, has realized the function of resource efficient scheduling and ordering management, for application of geographic information system to construct and run efficient laid a foundation.

\section{Acknowledgments}

At the point of finishing this paper, I'd like to express my sincere thanks to all those who have lent me hands in the course of my writing this paper. First of all, I'd like to take this opportunity to show my sincere gratitude to my college, Xiao Ming, who has given me so much useful advices on my writing. Secondly, I'd like to express my gratitude to my coauhor LiuFeng Tao, who offered me references and information on time. Last but not the least, I'd like to thank 
those leaders, teachers and working staff especially those in the China Three Gorges University.

\section{References}

[1] B Sun, H Wu, H Zhao, X Hu, "Research and application on plug-in technology in OpenSim", Audio Language and Image Processing (ICALIP), 2010 International Conference, 23-25 Nov. (2010), pp. 1392 $-1396$.

[2] Meng Wang, Yi Min Nie, Hao Yuan, "Research on the Urban Land Use Status and Potential Evaluation Information System Solution Based on OSGI Plug-in - A Case Study of Zhaoyuan, Shandong Province”, Advanced Materials Research, volumes 356 - 360, pp.2812-2815, (2011).

[3] Olga Levina, Vladimir Stantchev, "A Model and an Implementation Approach for Event-Driven Service Orientation”, International Journal On Advances in Software, volume 2, numbers 2 and 3, pp288-299, 2009.

[4] Jie Wang, Ming Yuan Jie, Bin Wu, "A Schema of Dynamic capability updating in BI-PAAS system”, Computer Science and Network Technology (ICCSNT), 2011 International Conference, 24-26 Dec. (2011), pp. 2556 - 2560.

[5] P. Laplante, A. Gold, and T. Costello, "Open Source Software: Is It Worth Converting?” IT Professional, vol. 9, no. 4, (2007), pp. 28-33.

[6] Balqies Sadoun and Omar Al-Bayari, "LBS and GIS Technology Combination and Applications" 1-4244-1031-2/07/@(2007) IEEE,pp. 578-583.

[7] Jaegeol Yim,Ilseok Ko,Ilseok Ko,"Implementation of a Prototype Positioning System for LBS on U-campus "Journal of Universal Computer Science, vol. 14, no. 14 (2008), 2381-2399

[8] Jingbin Liu, Ruizhi Chen, Ling Pei,Robert Guinness and Heidi Kuusniemi "A Hybrid Smartphone Indoor Positioning Solution for Mobile LBS ”Sensors (2012), 12, pp:17208-17233

[9] Feixiang CHEN, Chongjun YANG, Wenyang YU, Xiaoqiu LE, Jianyu YANG, "Research on Mobile GIS Based on LBS " 0-7803-90504/05/@(2005) IEEE pp:901-904

[10] C.M. Wang, Z.M. Wang and L.Z. Zhu,"Problems and Methods in the Location-Based Services,"Geomatics \& spacial information technology, vol. 32, June (2007), pp. 124-127.

[11] Xie, Y., et al. Indoor location technology and its applications base on improved LANDMARC algorithm. in Control and Decision Conference (CCDC), 2011 Chinese. 2011. Mianyang. 